\title{
In Vitro and In Vivo Evaluation of pH-Sensitive Hydrogels of Carboxymethyl Chitosan for Intestinal Delivery of Theophylline
}

\author{
Hemant Kumar Singh Yadav and H. G. Shivakumar \\ Department of Pharmaceutics, JSS College of Pharmacy, JSS University, Sri Shivarathreeshwara Nagar, Karnataka, \\ Mysore 570 015, India \\ Correspondence should be addressed to Hemant Kumar Singh Yadav, haisunny2@yahoo.co.in
}

Received 19 March 2012; Accepted 29 April 2012

Academic Editors: R. Cao, R. Gozalbes, and P.-W. Hsieh

Copyright ( $) 2012$ H. Kumar Singh Yadav and H. G. Shivakumar. This is an open access article distributed under the Creative Commons Attribution License, which permits unrestricted use, distribution, and reproduction in any medium, provided the original work is properly cited.

\begin{abstract}
Chitosan is a natural polymer which has limited solubility. Chitosan gets solubilized at acidic $\mathrm{pH}$ but is insoluble at basic pH. In the present study, carboxymethyl chitosan (CMC) was prepared which shows high swelling in basic $\mathrm{pH}$ and thus can delay the drug release and can act as matrix for extended release formulation. CMC was characterized by FTIR and NMR. pH-sensitive hydrogels of theophylline were formulated using CMC and carbopol 934. Hydrogels were evaluated for swelling, drug content in vitro drug release studies, and in vivo studies on rabbit. The swelling studies have shown little swelling in acidic pH $432 \%$ at the end of two hours and $1631 \%$ in basic $\mathrm{pH}$ at the end of 12 hours. The release profile of the formulation I containing CMC and carbopol in 1:1 ratio showed sustained release. In vivo studies showed that the release of theophylline from the prepared hydrogel formulation (Test) exhibit better prolonged action when compared to (standard) marketed sustained release formulation. The studies showed that the $\mathrm{pH}$-sensitive hydrogel of CMC can be used for extended release of theophylline in intestine and can be highly useful in treating symptoms of nocturnal asthma.
\end{abstract}

\section{Introduction}

Chitosan is biodegradable and biocompatible polymer. Since chitosan is insoluble in water, the use of chitosan in basic environment is limited and hence delivery of drugs to the intestine is not possible. A derivative of chitosan, that is $\mathrm{CMC}$, is soluble in water $[1,2]$. Amphoteric polyelectrolyte hydrogels possessing both positive and negative charges, and many researchers are using amphoteric polyelectrolyte hydrogels to develop controlled delivery systems such as an insulin pump for diabetics, matrices for molecular recognition or separation, and so forth. A lot of research is going on the stimuli-sensitive polymer hydrogels. Among stimuli-sensitive systems, $\mathrm{pH}$ or temperature-responsive hydrogels have been extensively studied in the biomedical field, because these two factors can be easily controlled and are applicable both in vitro and in vivo conditions $[3,4]$. $\mathrm{CMC}$ is amphoteric polyelectrolyte and has various applications due to its unique chemical, physical, and biological properties, especially its excellent biocompatibility. It is used to prepare wound dressings, artificial bone, and skin, is used as a bacteriostatic agent and blood anticoagulant also. It has also demonstrated good $\mathrm{pH}$ and ion sensitivity in aqueous solutions due to abundant $-\mathrm{COOH}$ and $-\mathrm{NH}_{2}$ groups [5]. Recent studies have shown that CMC has been used in preparation of nanoparticles for treatment of cancer $[6,7]$. The use of CMC has also been explored for delivery of antimicrobial agents [8] and proteins [9]. Extended release matrix tablets have been studied using chitosan and carbopol [10].

Authors of this work have previously investigated CMC hydrogels to deliver methylprednisolone [11]. They found that hydrogels show minimal swelling in acidic pH. Considering this behavior of hydrogels the present study was carried out. Carbopol 934 is a polymer which is sensitive to $\mathrm{pH}$ and was used in the present study along with chitosan. To combine the advantage of synthetic and natural polymers and at the same time maintain the property of natural polymers such as biodegradation and bioactivity, amphoteric polyelectrolyte hydrogels with $\mathrm{pH}$ sensitivity 
were synthesized with CMC and carbopol 934 in this work. The swelling behavior of the hydrogel under different $\mathrm{pH}$ was studied. The release behavior of theophylline was investigated, when it was loaded into the $\mathrm{pH}$-sensitive hydrogels. Theophylline is a antiasthmatic drug and the dosing of theophylline is complicated because it shows extensive variation in bioavailability among patients. About $75 \%$ of people with asthma have symptoms that disrupt both the length and depth of their nighttime sleep at least once a week. The number of inflammatory cells in the airways is highest in the early morning, with a peak at $4 \mathrm{AM}$. In one study, patients with nocturnal asthma were found to have a $20 \%$ decrease in lung function overnight compared with $4 \%$ in nonasthmatics. Hencem changing the timing or dosage of the medications may improve symptoms one experiences at night. Many different asthma medications have been specifically studied for their effectiveness at night.

Theophylline comes in both short-acting and slowrelease formulations, once or twice a day. It comes as a pill or in granules which should be swallowed whole, so as not to release too much medication at one time. The main drawback of this type of dosage form is that when blood levels are too high, unpleasant side effects may occur, such as nausea, vomiting, abdominal pain, jitteriness, insomnia, rapid or irregular heartbeat. Theophylline, if delivered to the intestine can be useful in treatment of nocturnal asthma and a single dose of a slow release or extended release theophylline preparation given at night, may provide effective control of nocturnal asthma symptoms.

In the present paper, an attempt was made to formulate a $\mathrm{pH}$-sensitive hydrogel from CMC containing theophylline which has not been attempted so far and evaluate it in vitro and in vivo. The polymer exhibits $\mathrm{pH}$ dependent swelling that is, they swell and release the drug depending on $\mathrm{pH}$ range, hence sustained or extended drug delivery is possible in the basic environment of gastrointestinal tract. CMC since soluble in water, undergoes extensive swelling in basic $\mathrm{pH}$ compared to acidic $\mathrm{pH}$ and drug release is maximum in intestine, thus it can be highly helpful in controlling symptoms of nocturnal asthma.

\section{Experimental}

Theophylline was gift sample from Strides Arco lab Limited, Bangalore. Chitosan (MW $=3.5 \times 10^{5},>80 \%$ deacetylated) was purchased from Sigma Aldrich, USA. Carbopol 934 was purchased from Loba Chemie Pvt. Ltd., India. All other chemicals were of analytical grade. There is no conflict of interests for any financial gain, as the chemicals were purchased from the companies.

2.1. Preparation of CMC. Chitosan solution was prepared in acetic acid and methanol, and acetic anhydride was added under stirring at room temperature. The mixture was stored overnight at room temperature to give a rigid gel. The prepared gel was agitated with $0.5 \mathrm{M} \mathrm{NaOH}$ in ethanol at room temperature overnight. The solution was precipitated by addition of concentrated $\mathrm{NH}_{4} \mathrm{OH}$ solution and filtered.
TABLE 1: Different formulation prepared of theophylline hydrogels.

\begin{tabular}{lcccccc}
\hline Sl. & Ingredient & F1 & F2 & F3 & F4 & F5 \\
No & $\downarrow$ Ratio $\rightarrow$ & $1: 1$ & $1.25: 1$ & $1.5: 1$ & $1.75: 1$ & $2: 1$ \\
\hline 1 & $\begin{array}{c}\text { Theophylline }(\mathrm{mg}) \\
\text { Carboxymethylchitosan } \\
\text { (mg) }\end{array}$ & 300 & 300 & 300 & 300 & 300 \\
2 & 1000 & 1250 & 1500 & 1750 & 2000 \\
3 & Carbopol 934 (mg) & 1000 & 1000 & 1000 & 1000 & 1000 \\
4 & Distilled water (mL) & 25 & 25 & 25 & 25 & 25 \\
5 & 1.75 M Acetic acid (mL) & 25 & 25 & 25 & 25 & 25 \\
\hline
\end{tabular}

The product was washed with $75 \%$ ethanol and dried in dessicator. The product which is formed after drying is $\mathrm{N}$ acetyl chitosan. $\mathrm{N}$-acetyl chitosan was suspended in 50\% $\mathrm{NaOH}$ and kept at $-20^{\circ} \mathrm{C}$ overnight. The product was transferred to 2-propanol and chloroacetic acid was added in portions under stirring. After stirring at room temperature for $2 \mathrm{hr}$, the reaction mixture was heated to $60^{\circ} \mathrm{C}$ for another $2 \mathrm{hr}$. Dialysis was carried out against deionized water for 3 days; the product obtained was dried in dessicator. The dried product obtained was CMC [12-14].

2.2. Preparation of Hydrogel. CMC solution was prepared in distilled water under stirring at $5000 \mathrm{rpm}$ for $30 \mathrm{~min}$. Theophylline was added to CMC solution and the solution was stirred for $15 \mathrm{~min}$. Carbopol 934 dissolved in $1.75 \mathrm{M}$ acetic acid was added to CMC solution gradually under stirring. The turbid dispersion obtained was immediately poured into petri dish and kept overnight for cross linking at room temp. The hydrogel obtained was cut into $1 \mathrm{~cm} \times 1 \mathrm{~cm}$ pieces and dried for $24 \mathrm{hr}$ under vacuum. The dried hydrogel was crushed and passed through sieve \#60/85. The hydrogel particles retained on sieve \#85 were used for further studies. The formulation chart of different formulations is given in Table $1[11]$.

2.3. Fourier Transforms Infrared (FTIR) Spectral Analysis. The prepared hydrogel was subjected to FTIR analysis by $\mathrm{KBr}$ pellet method using fourier transform infrared (FTIR) spectrophotometer (Perkin Elmer, spectrum-100, Japan). This was employed to ascertain the compatibility of drugs with the excipient.

2.4. Scanning Electron Microscopy (SEM). SEM studies were carried out on hydrogel samples after coating with gold palladium on a scanning electron microscope, Joel SEM analysis instrument, Japan.

2.5. Differential Scanning Calorimetry. Differential scanning calorimetry was performed on a pure sample of theophylline and its formulation, using Shimadzu DSC-50 apparatus. Differential scanning calorimetric thermograms of 2 to $3 \mathrm{mg}$ samples were recorded at a heating rate of $5^{\circ} \mathrm{C} / \mathrm{min}$ in an open aluminium pan over the range of $25^{\circ} \mathrm{C}-300^{\circ} \mathrm{C}$. 
2.6. Nuclear Magnetic Resonance (NMR). Nuclear magnetic resonance studies were carried out on CMC to determine whether the conversion of chitosan has occurred to CMC using $\mathrm{C}^{13}$ NMR and using an NMR spectrometer (DSX-300, Bruker, India). The solid state (without solvent) NMR was done at $75 \mathrm{MHz}$.

2.7. Estimation of Theophylline Content of the Hydrogels. Here an amount of hydrogels containing $20 \mathrm{mg}$ of theophylline was placed in $7.4 \mathrm{pH}$ phosphate buffer solution, for 24 hours. In the $7.4 \mathrm{pH}$ phosphate buffer solution the hydrogels swell and the drug is released. At the end of 24 hours, amount of theophylline present in $7.4 \mathrm{pH}$ phosphate buffer is determined spectrophotometrically at $272 \mathrm{~nm}$. The method was validated for linearity, accuracy, and precision. The method obeyed Beer's law in the concentration range 2$14 \mu \mathrm{g} / \mathrm{mL}$.

2.8. Swelling Studies. The $\mathrm{pH}$-dependent swelling property of hydrogel was studied by immersing the dry hydrogels in aqueous solutions of the $\mathrm{pH} 1.2 \mathrm{HCl}$ buffer for $2 \mathrm{hr}$ and then in $\mathrm{pH} 7.4$ phosphate buffer for another $8 \mathrm{hr}$. After regular intervals of time, hydrogels were removed from the aqueous solution, excess surface water was removed with filter paper, weighed, and returned to the same container until equilibrium was observed $[13,15]$. The degree of swelling $\left(W_{t}\right)$ was calculated at different times by means of following equation:

$$
S=\frac{\text { (weight of swollen hydrogel }- \text { weight of dry hydrogel }) \times 100}{\text { weight of swollen hydrogel }} .
$$

2.9. In Vitro Drug Release Studies. In vitro drug release from the hydrogels was carried out in triplicate at $37 \pm 0.1^{\circ} \mathrm{C}$ in USP XXII dissolution apparatus type II (six basket dissolution tester-USP XXII,TDT-08L, Electrolab, Mumbai, India) at a rotation speed of $50 \mathrm{rpm}$. A sample of hydrogel equivalent to $300 \mathrm{mg}$ of theophylline was used in each test. Drug release from the hydrogel was studied in $900 \mathrm{~mL}$ of dissolution medium $(2 \mathrm{hr}$ in $\mathrm{pH} 1.2 \mathrm{HCl}$ buffer and $10 \mathrm{hr}$ in $\mathrm{pH} 7.4$ phosphate buffer). Sample of dissolution fluid was withdrawn through a filter $(0.45) \mu \mathrm{m}$ at every hour and was assayed at $272 \mathrm{~nm}$ for theophylline content using a Shimadzu UV-1700 double beam spectrophotometer [13]. The release data obtained were fitted into korsmeyer-peppas equation, $\log \% R=\log K+n \log t$, where $R$ is the amount of drug released in given time $t, K$ is the release rate constant, and $n$ is the time exponent. A graph of $\log \% R \mathrm{v} / \mathrm{s} \log t$ was plotted. The intercept on $Y$-axis gave the value of $K$, the release rate constant and the slope the value of $n$, the time exponent. To determine the release mechanism, the parameter $n$ and $k$ were used.

2.10. In Vitro Wash-Off Test for Mucoadhesion. About 50 hydrogel particles were taken for the study and spread over the sheep's intestinal mucosa $(2 \times 2 \mathrm{~cm})$ taken as a biological substrate for studying mucoadhesive nature of the hydrogels. The prepared hydrogel was passed through sieve number 20; the particles which were retained on the sieve were coarser and were counted and taken for the study. The instrument designed was, disintegration apparatus USP, the 6 tubes were removed and the mocosa was fixed to the base of the apparatus. The medium chosen was $7.4 \mathrm{pH}$ phosphate buffer, every 5 min interval hydrogel particles adhering to the mucosa were counted. The study was carried out for $30 \mathrm{~min}$ [16].

2.11. In Vivo Studies. The in vivo release studies were conducted on albino rabbits weighing $2.5-3 \mathrm{~kg}$. The animals were divided into two groups of 6 rabbits each as a standard and the other as test. A written approval was obtained from the Institutional ethical committee of JSS Medical College and Hospital and JSS College of Pharmacy, Mysore, India. Detailed verbal and written information on the study was provided to the Veterinary Surgeon, Central Animal Facility, JSS Medical College and Hospital and permission was obtained. The animals were fasted for 12 hours before the capsules were introduced into the oesophagus and washed using $5 \mathrm{~mL}$ of distilled water in order to avoid the possible damage caused by chewing. Blood samples were collected from ear vein at 1, 2, 4, 8, 16, $24 \mathrm{hr}$ after the oral administration [17].

The blood samples were centrifuged and plasma was stored at $-20^{\circ} \mathrm{C}$ for further analytical determination. To the above samples, isopropyl alcohol was added and vortexed for $30 \mathrm{sec}$. The drug was extracted with $2 \mathrm{~mL}$ of chloroform and vortexed at high speed for $1 \mathrm{~min}$. After centrifugation at $1000 \mathrm{rpm}$ for $5 \mathrm{~min}$, the organic layer was evaporated and the residue was reconstituted with $100 \mathrm{~mL}$ of the mobile phase. This solution was injected into the HPLC system for analysis. The instrument used was Shimadzu LC-2010AHT. Acetonitrile $(7.5 \%)$ in $0.2 \%$ acetic acid solution was used as the mobile phase. Column used was $\mathrm{C}_{18}$. The temperature was kept ambient. Injection volume was $20 \mu \mathrm{L}$ at $1.5 \mathrm{~mL} / \mathrm{min}$ flow rate [18]. The sample run time was $8 \mathrm{~min}$.

The in vivo studies were conducted on prepared optimized hydrogel formulation (Test) and on marketed sustained release formulation Theobid SR tablet from Cipla (Standard).

2.12. Stability Studies. Stability studies were conducted on optimized formulation of CMC hydrogels to assess their stability with respect to their physical appearance, drug content, swelling, and drug release characteristics after storing them at $25^{\circ} \mathrm{C} /(\mathrm{RH}) 60 \%$, and $30^{\circ} \mathrm{C} /(\mathrm{RH}) 65 \%$ as per ICH $Q_{1} A\left(R_{2}\right)$ regulations for 6 months. 


\section{Results and Discussion}

3.1. Preparation of CMC. Chitosan is a unique polysaccharide derived from deacetylation of chitin. When chitosan is changed into O-carboxymethyl chitosan (O-CMC) by introducing $-\mathrm{CH}_{2} \mathrm{COOH}$ groups onto $-\mathrm{OH}$ along chitosan molecular chain, an amphoteric polyelectrolyte containing both cationic and anionic fixed charges was prepared [16]. By varying degree of deacetylation and carboxymethyl group substitution of the chitosan, we can obtain CMC. Carboxymethyl substituents were observed on amino and hydroxyl sites on the surface of modified chitosan. The preparation was carried out in two steps. First, N-acetyl chitosan was prepared using acetic anhydride, then carboxymethylation was done to get CMC. As given in literature at $50 \%$ concentration $\mathrm{NaOH}$ gives better degree of substitution, hence, $50 \%$ concentration was used [16]. The prepared $\mathrm{CMC}$ is white-colored free-flowing powder and shows good solubility in both water and organic solvents, which extends its range of applications. CMC shows characteristic behavior with $\mathrm{pH}$. This property and water solubility was used in preparing $\mathrm{pH}$-sensitive hydrogels in the present work.

3.2. Preparation of CMC Hydrogels. CMC is amphoteric in nature and contains positively charged groups, they interact with negatively charged carboxylic groups of Carbopol and form interpolyelectrolyte complexes (IPECs) which were stabilized by cooperative ionic bonds. Moreover, interpolymer interactions were possible between countercharged groups in the own macromolecule and of course between copolymer chains of different macromolecules. Due to its good solubility in wide range of $\mathrm{pH}$ values, the CMC solution could be readily blended with polyacrylic acid solution and homogenous hydrogels were obtained. The cross-linking was carried out at room temperature. Different formulations were prepared by varying the concentration of CMC by keeping the concentration of polyacrylic acid constant from F1-F5. For the formulation F1 to F5 the concentration of carboxymethylchitosan was increased gradually from $1 \%$, $1.25 \%, 1.5 \%, 1.75 \%$, and $2 \%$, respectively.

3.3. FTIR Studies. FTIR studies were carried out for carboxymethylchitosan and chitosan, and the spectra are given in Figure 1. Spectra showed signals of nonmodified chitosan at 1,653 and $1,560 \mathrm{~cm}^{-1}$ for the $\mathrm{C}-\mathrm{O}$ stretching (amide) and $\mathrm{N}-\mathrm{H}$ bending (amine), respectively. Other characteristic peaks of chitosan $\mathrm{O}-\mathrm{H}$ stretch, $\mathrm{C}-\mathrm{H}$ stretch, and $\mathrm{C}-\mathrm{O}$ stretch were present at 3,400-3,600, 2,800-2,900 and 1,020$1,180 \mathrm{~cm}^{-1}$, respectively. The spectra of carboxymethylchitosan are similar to that of the original chitosan with a new peak appearing at $1,703 \mathrm{~cm}^{-1}$, which is assigned to the carbonyl groups. This confirmed the conversion of chitosan to carboxymethylchitosan. Carboxymethylchitosan showed the disappearance of the $-\mathrm{NH}_{2}$ associated band at $1595 \mathrm{~cm}^{-1}$, which can be ascribed to characteristic vibration deformation of the primary amine $\mathrm{N}-\mathrm{H}$ and the appearance of some new intensive peaks at 2922-2852, 1466, and $721 \mathrm{~cm}^{-1}$ which can be attributed to the methyl groups and the long carbon segment of the quaternary ammonium salt. Characteristic peaks of the hydroxyl and second hydroxyl groups between 1152 and $1030 \mathrm{~cm}^{-1}$ did not change. Theophylline (pure drug) and hydrogel formulation were subjected for FTIR spectroscopic to ascertain whether there is any interaction between the drugs and polymers used. The characteristic peaks of the pure drug were compared with the peaks obtained for their formulation. It was observed that similar characteristic peaks appear with minor differences, at $1654 \mathrm{~cm}^{-1} \quad(\mathrm{C}=\mathrm{O}$ stretching amide $), 1596 \mathrm{~cm}^{-1} \quad(\mathrm{C}=\mathrm{C}$ stretching aromatic), $1307 \mathrm{~cm}^{-1}$ (C-O stretching) for theophylline and for the formulation as shown in Figure 2. Hence, it can be concluded that the drug is in free state and there is no interaction between drug and polymer used.

3.4. $C^{13}$ NMR Studies. The $C^{13}$ NMR of chitosan was studied as given in literature and compared with the spectra of CMC. Chitosan spectra show peaks at $177.9 \mathrm{ppm}$ and at $25 \mathrm{ppm}$, which are assigned to the carbonyl carbon of $-\mathrm{COCH} 3$ and the methyl carbon $(-\mathrm{CH} 3)$, respectively. These signals are less intense than the other signals. The signal at $101.3 \mathrm{ppm}$ is assigned to the hydrogen bonded to carbon of chitosan and the signals in $59.6 \mathrm{ppm}, 73.1 \mathrm{ppm}$, $81.1 \mathrm{ppm}, 78.6 \mathrm{ppm}$, and $64 \mathrm{ppm}$ are assigned to carbons of glucopyranose [19]. The $\mathrm{C}^{13} \mathrm{NMR}$ of carboxymethylchitosan was carried out and shown in Figure 3. Spectra show the signal shifted from $101.3 \mathrm{ppm}$ to $105.9 \mathrm{ppm}$ because of the electron-withdrawing effect of the carboxymethyl substituents. Since various different units occur in the structure of carboxymethylchitosan, many of the signals in the spectrum of chitosan appear split in the spectrum of carboxymethylchitosan. Thus, the signals at $60.1 \mathrm{ppm}$, $73.8 \mathrm{ppm}, 73.2 \mathrm{ppm}, 82.2 \mathrm{ppm} 78.2 \mathrm{ppm}$, and $63.9 \mathrm{ppm}$ are split and shifted in relation to those detected in the spectrum of the parent chitosan. The signal observed at $180.7 \mathrm{ppm}$ is assigned to the carbonyl carbons of carboxymethyl groups while the one detected at $177.9 \mathrm{ppm}$ corresponds to the carbonyl carbon of $-\mathrm{COCH} 3$ of the parent chitosan. The methylene groups $(-\mathrm{CH} 2)$, carbons give rise to the signals at 53 and $57.4 \mathrm{ppm}$, respectively. However, no signal was detected at $53 \mathrm{ppm}$ in the spectrum of carboxymethylchitosan Figure 3 and the weak signal at $58.4 \mathrm{ppm}$ can be probably assigned to the methylene $(-\mathrm{CH} 2)$ bonded to the amino group $(-\mathrm{NH})$ [19]. These features are taken as evidence that the carboxymethylation occurred at the hydroxyl as well as in the amino groups of chitosan which is also supported by FTIR studies. The spectra are shown in Figure 3.

3.5. Scanning Electron Microscopy. Scanning electron microscopy was carried out in order to study surface morphology, texture, and porosity of hydrogels. The SEM photograph of hydrogel clearly showed the rugged nature of hydrogel particles. The SEM photograph is shown in Figure 4.

3.6. Differential Scanning Calorimetry. DSC studies of pure drug and F1 were studied to determine the possible interaction between the drug and the hydrogel. Thermogram 


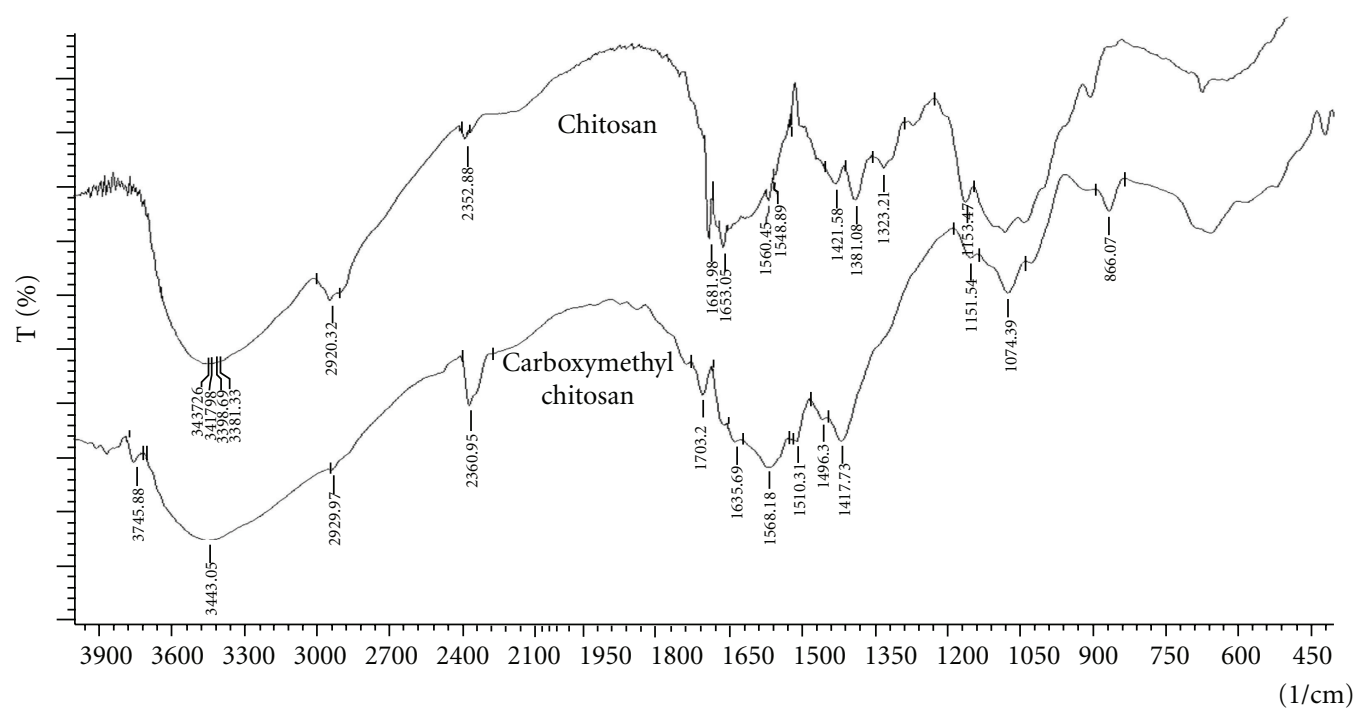

FIgURE 1: FTIR spectra of chitosan and CMC.

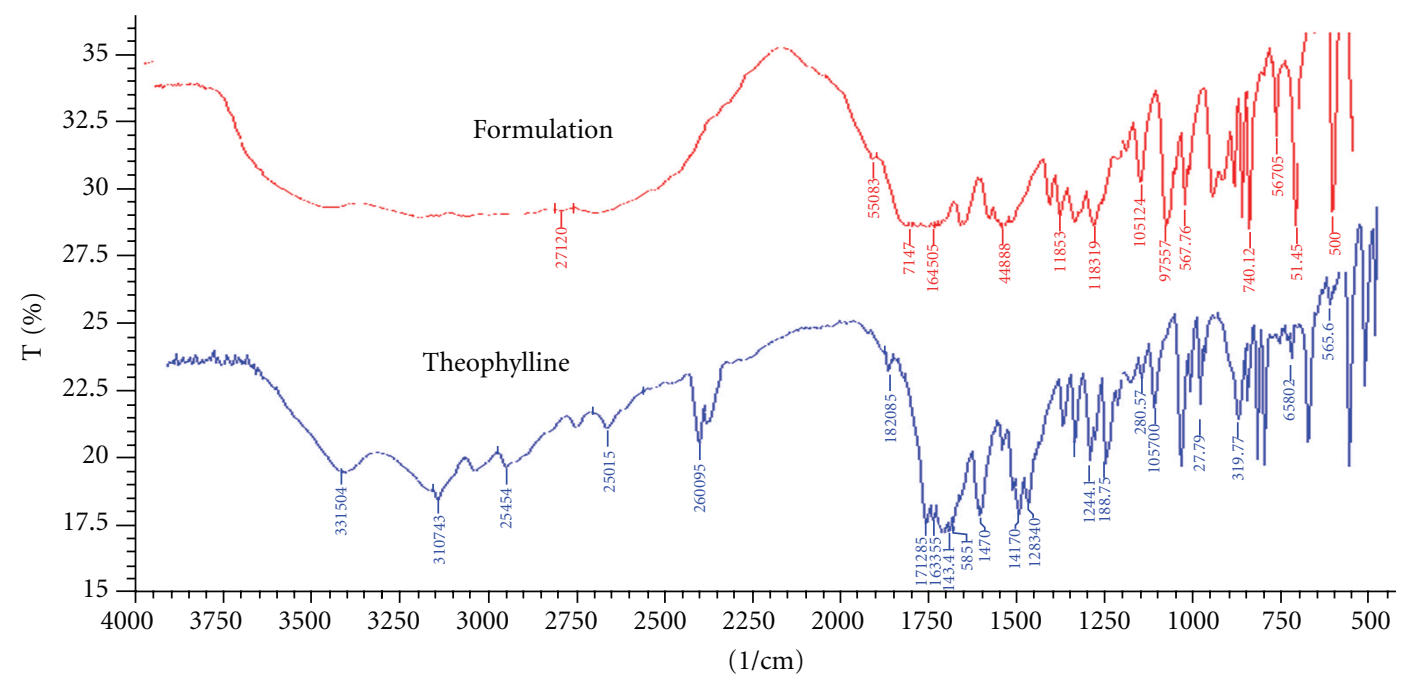

FIGURE 2: FTIR spectra of theophylline and hydrogel.

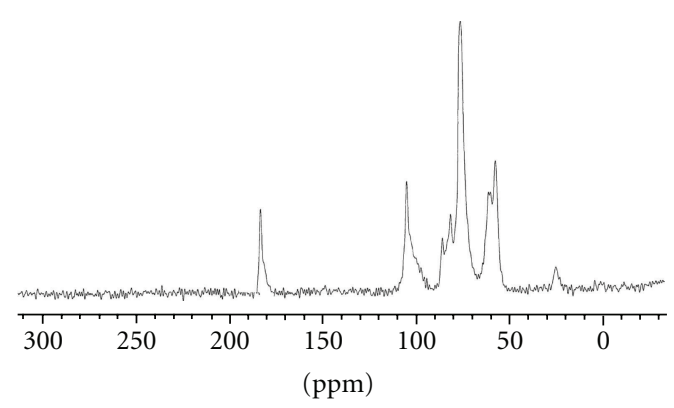

Figure 3: NMR spectra of CMC.

of theophylline has shown a sharp endothermic peak at $272.41^{\circ} \mathrm{C}$, which corresponds to its melting point. F1 formulation hydrogel also showed endothermic peaks at $271.59^{\circ} \mathrm{C}$. The evaluation of thermograms obtained from

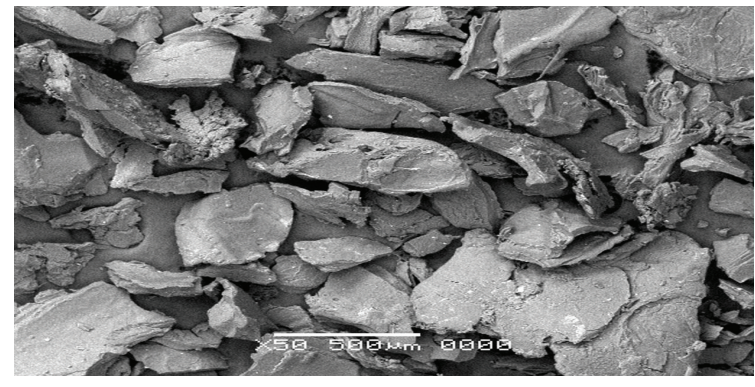

FIgURE 4: SEM photograph of hydrogel particles.

DSC revealed no interaction between the drug and polymers used, as there was no significant change in the melting point of theophylline. The obtained results are shown in Table 2. 
TABLE 2: DSC data of pure drug and F1 formulation.

\begin{tabular}{lcccc}
\hline Sl. No. & Drug and with hydrogel & $T_{0}\left({ }^{\circ} \mathrm{C}\right)$ & $T_{m}\left({ }^{\circ} \mathrm{C}\right)$ & $T_{c}\left({ }^{\circ} \mathrm{C}\right)$ \\
\hline $\mathbf{1}$ & Drug & 264.82 & 272.41 & 280.19 \\
$\mathbf{2}$ & F1 formulation & 267.41 & 272.09 & 279.66 \\
\hline
\end{tabular}

3.7. Drug Content. The test for drug content was carried out to ascertain that the amount of drug in the formulation. From the results obtained, it can be inferred that there is proper distribution of theophylline in the hydrogels. The drug content analysis showed that the drug is uniformly distributed in the range of $74.5-88.6 \%$ of the total amount of the drug added in different formulations.

3.8. Swelling Studies. The swelling behavior of CMC was studied. Swelling studies were conducted for $12 \mathrm{hrs}$ but swelling did not show significant change after $10 \mathrm{hrs}$ until $12 \mathrm{hrs}$ hence data for $10 \mathrm{hrs}$ is presented. The swelling in water mainly depends on the osmotic pressure difference between inside the gel and the surroundings caused by redistribution of mobile ions. The swelling was observed to be more at basic $\mathrm{pH}$ due to increase in the number of mobile ions inside the gel and large osmotic pressure leads to swelling. The results for swelling studies are shown in Figure 5. \% swelling was found in range of 432.75-1631.56. The results indicate that with an increase in $\mathrm{pH}$ from 1.2 to 7.4 , a considerable increase in swelling was observed for all the hydrogel formulations, which may be due to the dissociation of the $-\mathrm{COOH}$ groups of $\mathrm{CMC}$, thereby increasing the osmotic pressure inside the hydrogels resulting in increased swelling [16]. Swelling increased when ratio of CMC was changed till 1.5:1 with polyacrylic acid, that is, formulation F1-F3 but further increase in ratio, that is, 1.75: 1 and 2.0: 1 (F4 and F5) swelling decreased. This shows that $\mathrm{CMC}$ and polyacrylic acid have synergistic effect till certain ratio as a result of which swelling increases but further increase in CMC amount resulted in reduced water uptake which may be attributed to the antagonistic effect resulting in decrease swelling. Swelling strongly depends on the extent of cross-linking. At lower cross-linking, the network is loose with a greater hydrodynamic free volume, so that the chains can accommodate more of the solvent molecules resulting in higher swelling. In this study it was found that the swelling was increased when the $\mathrm{pH}$ was changed from acidic to basic, and it conforms that the prepared hydrogel was sensitive to $\mathrm{pH}$. The effect on swelling with time is shown is Figure 5.

3.9. In Vitro Drug Release Studies. The In vitro release studies were carried out for all the formulations in both acidic and basic media. The release studies were carried in the $\mathrm{pH}$ 1.2 $\mathrm{HCl}$ buffer for the first two hours, to mimic the acidic conditions prevailing in the stomach. For the next 10 hours, the release studies were carried out in $\mathrm{pH} 7.4 \mathrm{pH}$ phosphate buffer, to mimic the alkaline conditions of the intestine. For the initial 2 hours that is, in the $\mathrm{pH} 1.2 \mathrm{HCl}$ buffer, the percentage drug release was found to be low in all the cases; this can be attributed to the fact that the hydrogel swells

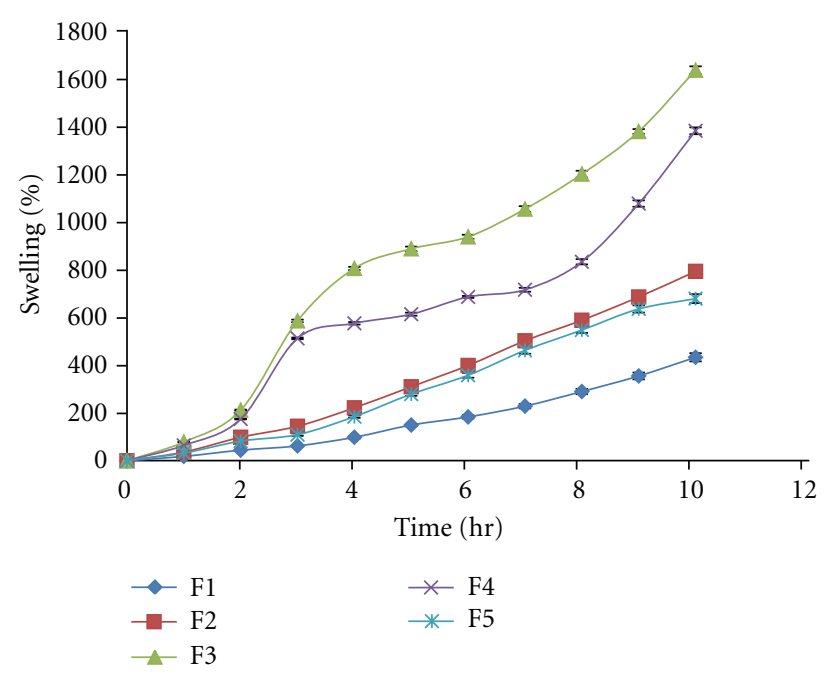

Figure 5: \% swelling of F1-F5 hydrogels for $10 \mathrm{hr}$.

less in the acidic medium. When the dissolution medium was changed to $\mathrm{pH} 7.4 \mathrm{pH}$ phosphate buffer, the release was found to increase, with time. The drug release showed effect on basis of swelling. Drug release decreased, from F1-F3 and again increased for F4-F5. The effect observed is based on swelling, as swelling increases the drug release decreases and again when swelling decreases drug release increases as shown in Figure 6. On the basis of above studies conducted F1 was chosen as optimized formulation as it showed desired sustained-release profile along with other drug content and swelling studies results. All formulations present an initial burst effect. It may be attributed to diffusion of the drug caused by rapid gel swelling and also the release of drug adsorbed towards the surface of the gel matrix. The $\%$ drug release was found in range of 37.21-98.47 at the end of $12 \mathrm{hrs}$. The data obtained from in vitro release studies was fitted into various mathematical models. The regression coefficient, $R^{2}$ obtained is given in Table 3. $R=$ regression coefficient. The value of $n$ determined from korsmeyer peppas equation was in the range of $0.5-0.7$, which indicates the drug release from the hydrogels followed non-fickian or anomalous mechanism (relaxation controlled).

3.10. In Vitro Wash-Off Test for Mucoadhesion. The mucoadhesive study showed that all the hydrogel particles get detached from the mucosa within $20 \mathrm{~min}$. This study shows that carboxymethylchitosan does not have a good mucoadhesion property when compared to well-known mucoadhesive strength of parent chitosan. This can be attributed to the better solubility of CMC in water and organic solvents.

3.11. In Vivo Studies. In vivo studies were carried out for Theobid SR tablet from Cipla (product A) and theophylline loaded hydrogels of CMC both containing $300 \mathrm{mg}$ of theophylline on albino rabbits. Blood samples were withdrawn at different time intervals and plasma concentrations of theophylline were estimated, the profile is presented in 
TABLE 3: Model fitting for different formulations.

\begin{tabular}{|c|c|c|c|c|c|}
\hline & \multicolumn{5}{|c|}{$R^{2}$} \\
\hline & $\mathrm{F} 1$ & $\mathrm{~F} 2$ & F3 & $\mathrm{F} 4$ & F5 \\
\hline Zero order & 0.763 & 0.682 & 0.714 & 0.701 & 0.670 \\
\hline First order & 0.861 & 0.737 & 0.752 & 0.737 & 0.00 \\
\hline Higuchi & 0.902 & 0.829 & 0.901 & 0.890 & 0.832 \\
\hline Peppas & 0.957 & 0.910 & 0.950 & 0.963 & 0.897 \\
\hline Best fit model & Peppas & Peppas & Peppas & Peppas & Peppas \\
\hline Diffusion exponent Peppas model & $n=0.6937$ & $n=0.5826$ & $n=0.784$ & $n=0.752$ & $n=0.652$ \\
\hline
\end{tabular}

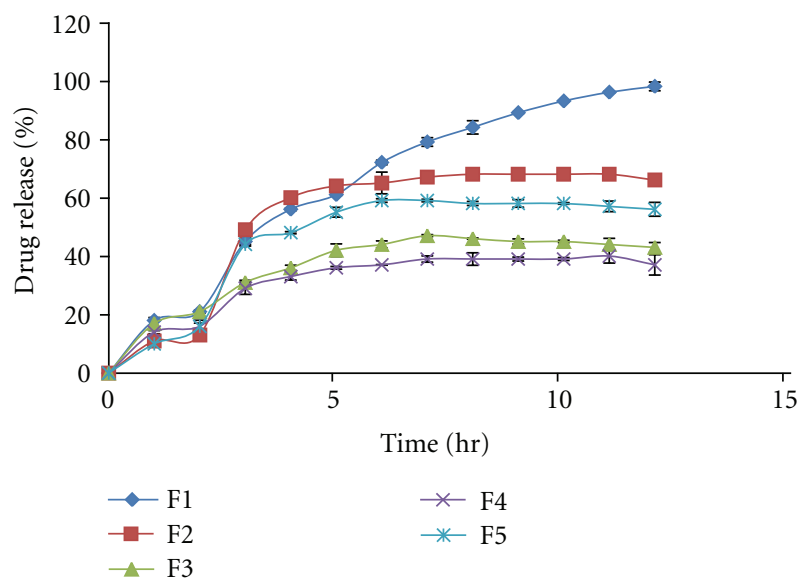

FIGURE 6: In vitro drug release of different formulations.

TABLE 4: A statistical comparison of the mean values of pharmacokinetic parameters of product A and B.

\begin{tabular}{lccc}
\hline Parameters & Product A & Product B & $P$ \\
\hline$C_{\max }$ & $12.34 \pm 2.42 \mu \mathrm{g} / \mathrm{mL}$ & $09.69 \pm 4.12 \mu \mathrm{g} / \mathrm{mL}$ & $<0.05$ \\
$T_{\max }$ & $5.0 \pm 0.81 \mathrm{hr}$ & $6.0 \pm 0.75 \mathrm{hr}$ & $<0.05$ \\
Kel & $0.08410 \mathrm{~h}^{-1}$ & $0.07813 \mathrm{~h}^{-1}$ & $<0.05$ \\
$t_{1 / 2}$ & $8.24 \pm 4.7 \mathrm{hrs}$ & $8.87 \pm 5.74 \mathrm{hrs}$ & $<0.05$ \\
AUC $_{0-24}$ & $101.73 \pm 16.5 \mu \mathrm{g} \cdot \mathrm{hr} / \mathrm{mL}$ & $123.17 \pm 21.5 \mu \mathrm{g} \cdot \mathrm{hr} / \mathrm{mL}$ & $<0.05$ \\
\hline
\end{tabular}

Figure 7. Statistical comparison of the mean values of pharmacokinetic parameters derived for both products A and B are given in Table 4. From the data obtained, it may be observed that after oral administration, peak plasma concentration $C_{\max }$ of $12.34 \pm 2.42 \mu \mathrm{g} / \mathrm{mL}$ was observed for product $A$ and $09.69 \pm 4.12 \mu \mathrm{g} / \mathrm{mL}$ for product B. From the comparison of mean values of plasma concentrations of product $\mathrm{A}$ and $\mathrm{B}$, it was observed that product $\mathrm{B}$ has lower plasma concentrations. It was observed that the plasma concentration of theophylline in all animals after $24 \mathrm{hrs}$ of oral administration was below $20 \mu \mathrm{g} / \mathrm{mL}$ for both products. It was also observed from the studies that the therapeutic concentration range of theophylline maintained for about 24 hrs following a single oral dose administration for both products.

From the data obtained, it may be observed that the time taken to reach peak plasma concentration $T_{\max }$ was

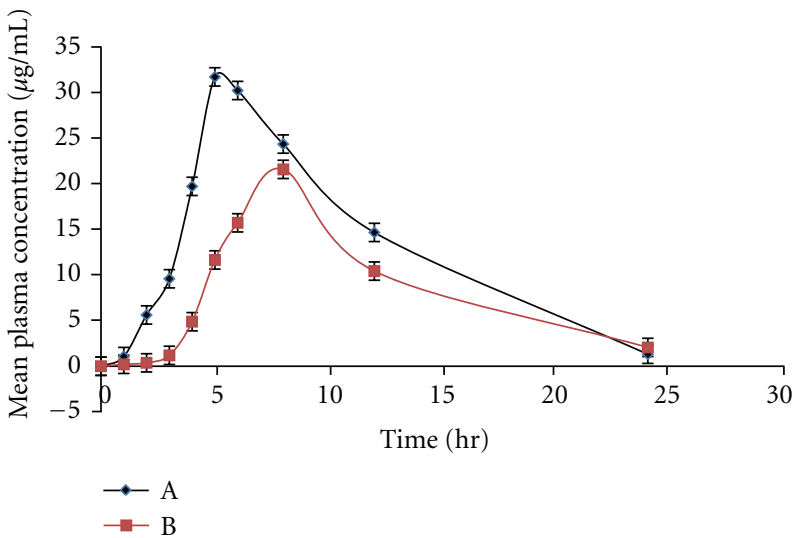

FIGURE 7: Mean plasma concentrations time profiles of theophylline product $\mathrm{A}$ and $\mathrm{B}$.

$5.0 \pm 0.81 \mathrm{hrs}$ for product $\mathrm{A}$ and $6.0 \pm 0.75 \mathrm{hrs}$ for product B. Mean elimination rate constant $\mathrm{Kel}$ was found to be $0.08410 \mathrm{~h}^{-1}$ for product A and $0.07813 \mathrm{~h}^{-1}$ for product $\mathrm{B}$. Similarly mean elimination half life $t_{1 / 2}$ for product A was $8.24 \pm 4.7 \mathrm{hrs}$ and for product B $8.87 \pm 5.74 \mathrm{hrs}$. The mean $\mathrm{AUC}_{0-24}$ values for product A was $101.73 \pm 16.5 \mu \mathrm{g} \cdot \mathrm{hr} / \mathrm{mL}$ and for product B $123.17 \pm 21.5 \mu \mathrm{g} \cdot \mathrm{hr} / \mathrm{mL}$. The lower $C_{\max }$, prolonged $T_{\max }$, of theophylline in rabbits indicated that the drug release from the product $B$ is slow, thereby providing a prolonged and controlled in vivo delivery of the drug. These in vivo absorption characteristics are in confirmation with the observed in vitro drug release rate of the drug from the hydrogel.

3.12. Stability Studies. Stability studies were conducted for different formulations for a period of 6 months. At specific time intervals, the samples were tested for drug content, swelling, and \% drug release. The drug content in samples was tested at 2 different conditions along with 95\% confidence limits, using sigma plot software 10.0 as shown in Figures 8 and 9. Stability studies results obtained at various intervals showed that the hydrogel prepared from CMC did not show significant difference in physical appearance at the end of 6 months except in change of colour from light brown to brown. \% drug release, \% swelling, and mucoadhesion did not change significantly at the end of stability studies. From the data, it can be seen that hydrogels prepared are stable at given two conditions. 


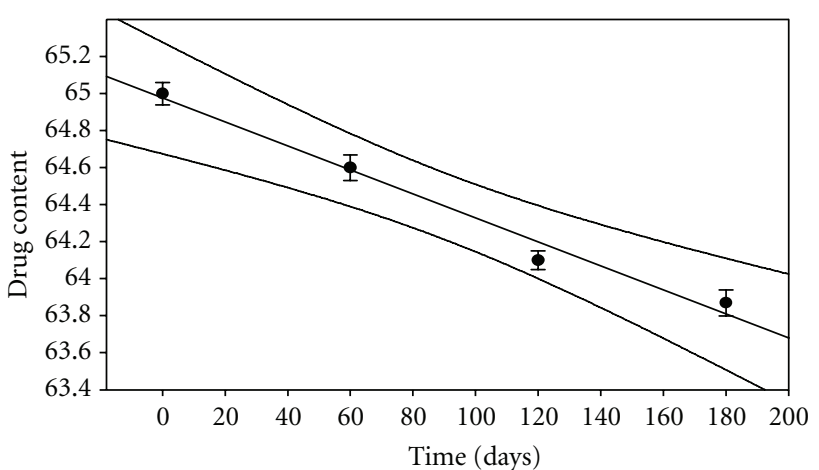

- Theophylline content

Regression line $95 \%$ conf. int.

Figure 8: Theophylline content at $25^{\circ} \mathrm{C} /(\mathrm{RH}) 60 \%$.

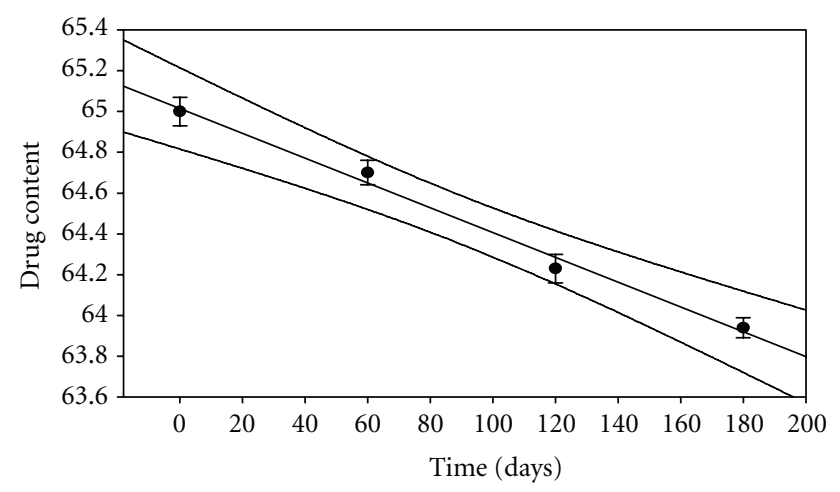

- Theophylline content Regression line - $95 \%$ conf. int.

Figure 9: Theophylline content at $30^{\circ} \mathrm{C} /(\mathrm{RH}) 65 \%$.

\section{Conclusion}

From the results obtained, it may be concluded that the prepared hydrogels were $\mathrm{pH}$-sensitive and the degree of swelling of hydrogel depends on the concentration of crosslinking agent as well as on the $\mathrm{pH}$ of the environment. As the theophylline is released highly in basic $\mathrm{pH}$ the peak theophylline concentration will be achieved at early morning which will be highly beneficial to the patients suffering from nocturnal asthma. The in vitro and in vivo studies showed that the drug release is slowed down and prolonged which is better than commercial available formulation and hence better. Thus, the hydrogel prepared using CMC can be used to deliver theophylline in sustained manner and can be used effectively against nocturnal asthma; CMC can also be useful for the delivery of drugs which are unstable in acidic $\mathrm{pH}$.

\section{Acknowledgments}

The authors thank the JSS Mahavidyapeetha, Mysore and JSS University, Mysore for their valuable support to carry out this research.

\section{References}

[1] R. Kumar and N. V. Majeti, "A review of chitin and chitosan applications," Reactive and Functional Polymers, vol. 46, no. 1, pp. 1-27, 2000.

[2] M. Amiji and R. Hejazi, "Chitosan-based gastrointestinal delivery systems," Journal of Controlled Release, vol. 89, no. 2, pp. 151-165, 2003.

[3] A. S. Hoffman, "Hydrogels for biomedical applications," Advanced Drug Delivery Reviews, vol. 54, no. 1, pp. 3-12, 2002.

[4] B. Guo, J. Yuan, L. Yao, and Q. Gao, "Preparation and release profiles of $\mathrm{pH} /$ temperature-responsive carboxymethyl chitosan/P(2-(dimethylamino) ethyl methacrylate) semi-IPN amphoteric hydrogel," Colloid and Polymer Science, vol. 285, no. 6, pp. 665-671, 2007.

[5] X. G. Chen, Z. Wang, W. S. Liu, and H. J. Park, "The effect of carboxymethyl-chitosan on proliferation and collagen secretion of normal and keloid skin fibroblasts," Biomaterials, vol. 23, no. 23, pp. 4609-4614, 2002.

[6] M. E. Mathew, J. C. Mohan, K. Manzoor, S. V. Nair, H. Tamura, and R. Jayakumar, "Folate conjugated carboxymethyl chitosan-manganese doped zinc sulphide nanoparticles for targeted drug delivery and imaging of cancer cells," Carbohydrate Polymers, vol. 80, no. 2, pp. 442-448, 2010.

[7] A. Dev, J. C. Mohan, V. Sreeja et al., "Novel carboxymethyl chitin nanoparticles for cancer drug delivery applications," Carbohydrate Polymers, vol. 79, no. 4, pp. 1073-1079, 2010.

[8] X. Wang, B. Liu, J. Ren et al., "Preparation and characterization of new quaternized carboxymethyl chitosan/rectorite nanocomposite," Composites Science and Technology, vol. 70, no. 7, pp. 1161-1167, 2010.

[9] I. M. El-Sherbiny, "Enhanced pH-responsive carrier system based on alginate and chemically modified carboxymethyl chitosan for oral delivery of protein drugs: preparation and in-vitro assessment," Carbohydrate Polymers, vol. 80, no. 4, pp. 1125-1136, 2010.

[10] S. H. Park, M. K. Chun, and H. K. Choi, "Preparation of an extended-release matrix tablet using chitosan/Carbopol interpolymer complex," International Journal of Pharmaceutics, vol. 347, no. 1-2, pp. 39-44, 2008.

[11] G. B. Joshi, H. Ksy, M. N. Singh, and H. G. Shivakumar, "Development of $\mathrm{pH}$ sensitive hydrogel for intestinal delivery of methyl prednisolone using novel chitosan derivative," International Journal of Pharmacy and Pharmaceutical Sciences, vol. 3, no. 1, pp. 200-203, 2011.

[12] X. G. Chen and H. J. Park, "Chemical characteristics of Ocarboxymethyl chitosans related to the preparation conditions," Carbohydrate Polymers, vol. 53, no. 4, pp. 355-359, 2003.

[13] Y. H. Lin, H. F. Liang, C. K. Chung, M. C. Chen, and H. W. Sung, "Physically crosslinked alginate/N,O-carboxymethyl chitosan hydrogels with calcium for oral delivery of protein drugs," Biomaterials, vol. 26, no. 14, pp. 2105-2113, 2005.

[14] A. J. Dong and M. H. Feng, "Synthesis and properties of Ocarboxymethyl chitosan/methoxypoly(ethylene glycol) graft copolymers," Journal of Materials Science, vol. 19, pp. 869-876, 2008.

[15] L. Chen, Z. Tian, and Y. Du, "Synthesis and $\mathrm{pH}$ sensitivity of carboxymethyl chitosan-based polyampholyte hydrogels for protein carrier matrices," Biomaterials, vol. 25, no. 17, pp. 3725-3732, 2004.

[16] K. P. R. Chowdary and Y. Srinivasa Rao, "Preparation and evaluation of mucoadhesive microcapsules of indomethacin," Indian Journal of Pharmaceutical Sciences, vol. 65, no. 1, pp. 49-52, 2003. 
[17] Z. Muskó, K. Pintye-Hódi, R. Gáspár et al., "Study of in vitro and in vivo dissolution of theophylline from film-coated pellets," European Journal of Pharmaceutics and Biopharmaceutics, vol. 51, no. 2, pp. 143-146, 2001.

[18] P. Om, R. Rajat, and P. D. Sethi, Quantitative Analysis of Drugs in Pharmaceutical Formulation, CBS Publishers \& Distributors, New Delhi, India, 3rd edition, 2008.

[19] S. Abreu, F. Campana, and R. Fernanda, "Preparation and characterization of carboxymethyl chitosan. Polímeros," Ciência e Tecnologia, vol. 15, pp. 79-83, 2005. 

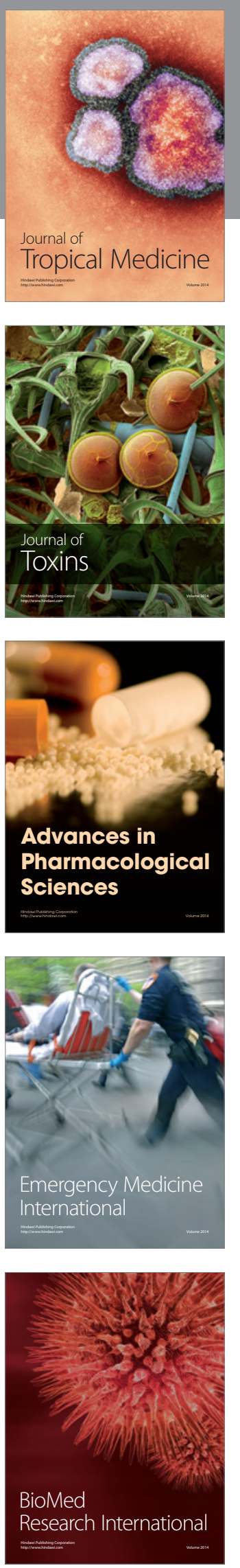
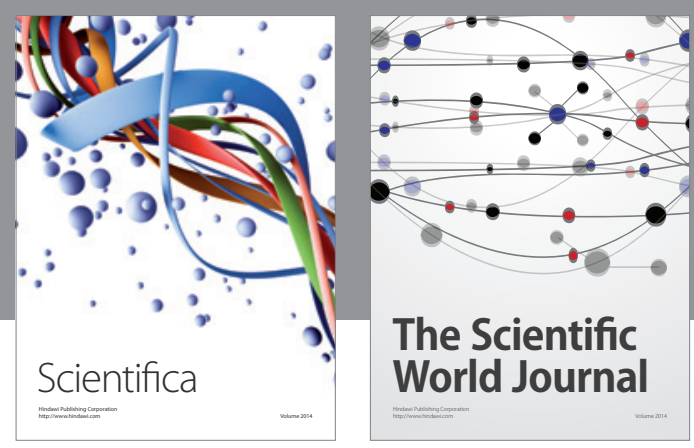

The Scientific World Journal
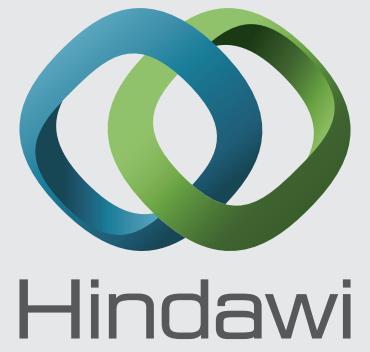

Submit your manuscripts at

http://www.hindawi.com
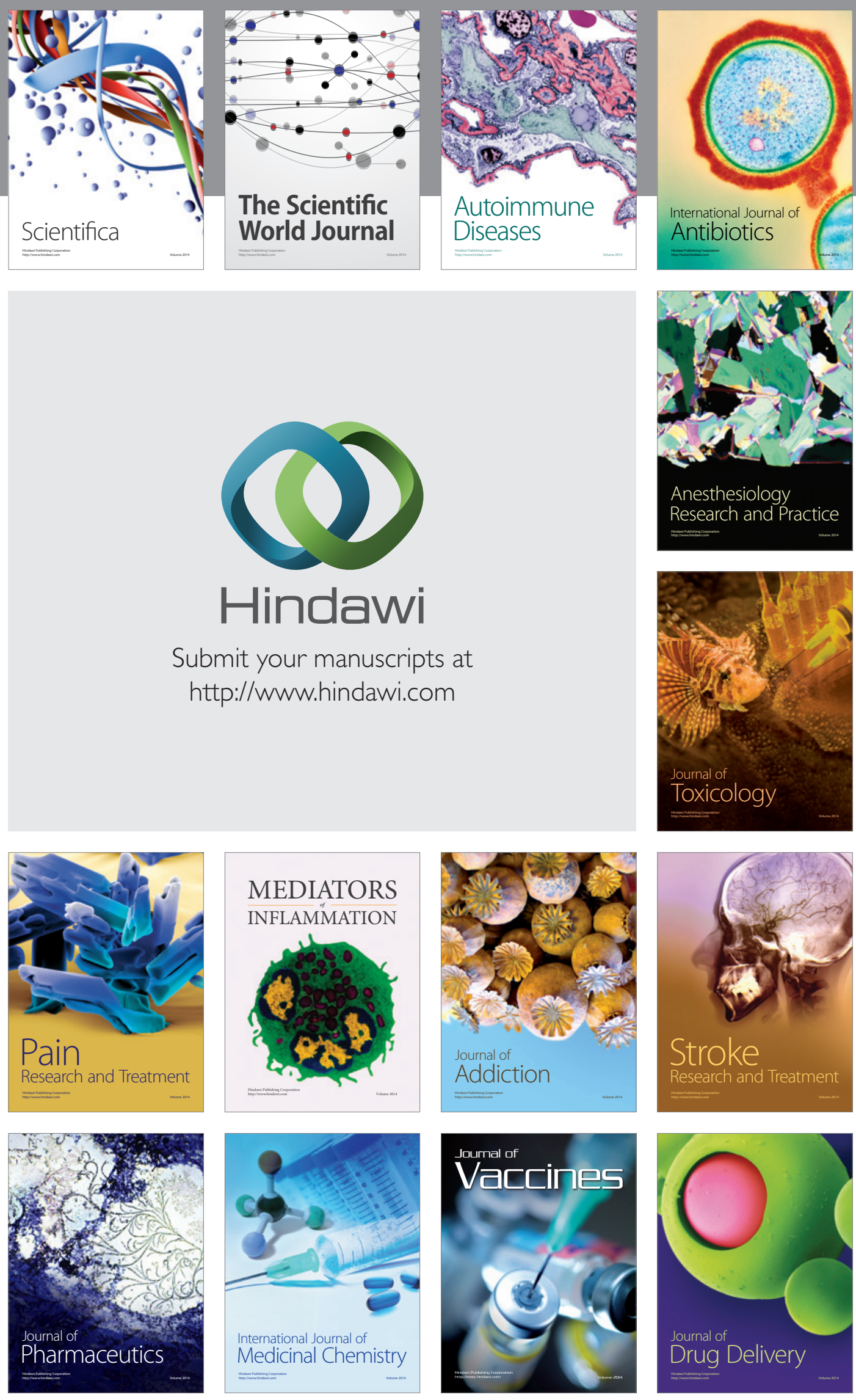